\title{
Sub-chronic administration of doxorubicin to Wistar rats results in oxidative stress and unaltered apoptotic signaling in the lung
}

\author{
Nuno G. Machado a , Inês Baldeiras ${ }^{b, c}$, Gonçalo C. Pereira ${ }^{a}$, Susana P. Pereira ${ }^{a}$, Paulo J. Oliveira ${ }^{a, *}$ \\ a Center for Neurosciences and Cell Biology, Department of Life Sciences, University of Coimbra, Portugal \\ b Laboratory of Neurochemistry, Department of Neurology, Coimbra University Hospital, Portugal \\ ${ }^{\mathrm{c}}$ Center for Neuroscience and Cell Biology, Faculty of Medicine, University of Coimbra, Portugal
}

\section{A R T I C L E I N F O}

\section{Article history:}

Received 19 May 2010

Received in revised form

27 September 2010

Accepted 28 September 2010

Available online 7 October 2010

\section{Keywords:}

Doxorubicin

Lung toxicity

Mitochondria

Apoptosis

Oxidative stress

\begin{abstract}
A B S T R A C T
Despite the vast published data on cardiac toxicity, there is still little work done regarding the toxicity of the antineoplastic agent Doxorubicin (DOX) in the lung. The aim of the present work was to determine if DOX causes alterations in selected apoptotic proteins and oxidative stress in the lung, in a similar manner to what occurs in the heart. For that purpose, lungs from Wistar-Han rats sub-chronically treated with vehicle or DOX for seven weeks were collected and analyzed concerning several proteins involved in mitochondrial permeabilization and apoptotic pathways, including p53, Bax and Bcl-2 and different oxidative stress markers. After sub-chronic DOX treatment, no alterations in lung proteins involved in mitochondrial membrane permeabilization or caspase 3 and 9-like activities were found. Nevertheless, an increase in malondialdehyde levels and a decrease in the lung concentration of vitamin E were detected, despite no alterations in reduced and oxidized glutathione. The results obtained indicate for the first time that lungs from DOX-treated rats appear to be susceptible to increased lipid peroxidation, which can explain some cases of DOX-induced lung toxicity.
\end{abstract}

(c) 2010 Elsevier Ireland Ltd. All rights reserved.

\section{Introduction}

Anthracycline antibiotics are among the most effective and commonly used anticancer drugs [1], with the most classic example being Doxorubicin (DOX), commonly used against ovarian, breast, lung, uterine and cervical cancers, Hodgkin's disease, soft tissue and primary bone sarcomas, as well against several other cancer types [2]. However, an irreversible [3] and dose-dependent cardiotoxicity often occurs in patients treated with DOX, which limits the clinical application of the drug [4]. DOX-induced cardiotoxicity has been attributed to a number of factors including the formation of reactive oxygen species (ROS), inhibition of the expression of cardiomyocyte-specific genes and altered molecular signaling pathways [5]. Augmented apoptotic signaling has also been considered an important contributing factor in DOX cardiotoxicity [6-9]. Several apoptotic end-points have been measured in patients, laboratory animals and cardiac cells treated with DOX [10], including DNA laddering and increase in the number of apoptotic nuclei [11], DNA fragmentation and caspase 3 activation [12] or cell membrane blebbing [13], among others. Other alterations have been described to accompany DOX-induced toxicity, including interference with

\footnotetext{
* Corresponding author. Tel.: +351 239 855760; fax: +351 239855789

E-mail address: pauloliv@ci.uc.pt (P.J. Oliveira).
}

mitochondrial calcium homeostasis at subclinical cumulative doses [14-16]. DOX-induced mitochondrial calcium deregulation was associated with induction of the mitochondrial permeability transition (MPT) [17,18]. As described, augmented oxidative stress is among one of the several hypotheses [19] that explain the mechanism and consequences of DOX cardiotoxicity. One particular important role has been attributed to mitochondrial complex I [20] which transfers electrons to DOX, generating a reactive semiquinone radical in the process [21]. Several authors have described already DOX-induced oxidative damage to mitochondrial membranes [22] and proteins [23], accompanied by a decrease in cellular energy charge [24], showing evidence that mitochondria are one important mediator in the development of DOX toxicity.

Despite the well-known side effects of DOX in the heart, little is known about the lung, although several cases of DOX lung toxicity have already been reported. DOX-induced toxicity can cause a range of pulmonary complications such as bronchospasm, local edema, interstitial pneumonitis and pulmonary fibrosis $[25,26]$. Moreover, pulmonary inflammation also results from prolonged exposure of the lung tissue to DOX [27]. Nevertheless, no studies in the literature describing DOX effects in the lung tissue with regard to oxidative stress and the appearance of apoptotic markers are available. Therefore, the purpose of this work was to investigate if DOX sub-chronic treatment would result in triggering of apoptotic signaling as well as increased oxidative stress in the lung. 


\section{Materials and methods}

\subsection{Materials}

Doxorubicin was obtained from Sigma (Barcelona, Spain). For Western blotting, primary antibodies used were goat anti-p53 (Sc6243-G) and goat anti-adenine nucleotide translocator (ANT) (Sc9300) from Santa Cruz Biotechnology, Inc. (San Diego, CA, USA); rabbit anti-B-cell leukemia/lymphoma 2 protein (Bcl-2) (\#2870), rabbit anti-BCL-2-associated X protein (Bax) (\#2772), and rabbit anti-cytochrome c oxidase subunit IV (COX IV) (\#4844) from Cell Signaling (Danvers, MA, USA); rabbit anti-voltage-dependent anion channel (VDAC/porin) (ab34726) and mouse anti-NADH dehydrogenase (ubiquinone) Fe-S protein 3 (NDUFS3) (ab14711) from Abcam (Cambridge, UK); rabbit anti-dinitrophenylhydrazine (DNP) (D9656) and mouse anti- $\beta$-actin (A5441) from Sigma (Barcelona, Spain). The secondary antibodies used were goat antirabbit IgG-AP (sc-2007), rabbit anti-goat IgG-AP (sc-2771), and goat anti-mouse IgG-AP (sc-2008), all from Santa Cruz Biotechnology, Inc. Molecular weight markers proteins were obtained from Bio-Rad Laboratories, Inc. (Hercules, CA, USA), while the ECF substrate was obtained from GE Healthcare Life Sciences (Buckinghamshire, UK). Caspase substrates were obtained from Calbiochem, Merck Chemicals Ltd. (Nottingham, UK). All the remaining reagents and chemical compounds used were of the greatest degree of purity commercially available. In the preparation of every solution, ultrapure distilled water, filtered by the Milli $Q$ from a Millipore system, was always used (conductivity $<18 \mu \mathrm{S} \mathrm{cm}^{-1}$ ) in order to minimize as much as possible contamination with metal ions.

\subsection{Preparation of the biological material}

Male Wistar-Han rats (8-10 animals per group, eight weeks of age at the beginning of DOX treatment) were housed in our credited animal colony (Laboratory Research Center, Faculty of Medicine, University of Coimbra). Animals were group-housed in type III-H cages (Tecniplast, Italy) and maintained in specific environmental requirements $\left(22^{\circ} \mathrm{C}, 45-65 \%\right.$ humidity, $15-20$ changes/hour ventilation, $12 \mathrm{~h}$ artificial light/dark cycle, noise level $<55 \mathrm{~dB}$ ) and free access to standard rodent food (4RF21 GLP certificate, Mucedola, Italy) and acidified water (at $\mathrm{pH} 2.6$ with $\mathrm{HCl}$ to avoid bacterial contamination). Rats were treated with weekly subcutaneous injections of vehicle (sc, saline solution, $\mathrm{NaCl} 0.9 \%$ ) or DOX (sc, $2 \mathrm{mg} \mathrm{kg}^{-1}$ ) during seven weeks to a total dosage of $14 \mathrm{mg} \mathrm{kg}^{-1}$. Animals were sacrificed one week after the last injection. This sub-chronic treatment protocol was demonstrated by our group to induce cardiac mitochondrial toxicity in rats [16]. In adherence to procedures approved by the Institutional Animal Care and Use Committee, the animals were euthanatized by cervical displacement and decapitation. Body and lung weights were registered at the day of the sacrifice. After sacrifice, the lungs were immediately removed, with blood washed out in phosphate buffer saline (PBS) solution, and subsequently frozen at $-80^{\circ} \mathrm{C}$ for future analyses. For experiments, lung tissue was washed with PBS and incubated for $30 \mathrm{~min}$ on ice with $100 \mathrm{ml}$ of lysis buffer $(10 \mathrm{mM}$ Tris- $\mathrm{HCl}$, $10 \mathrm{mM} \mathrm{NaH} \mathrm{PO}_{4} / \mathrm{NaHPO}_{4}, \mathrm{pH} 7.5,130 \mathrm{mM} \mathrm{NaCl}, 1 \%$ Triton X-100, and $10 \mathrm{mM}$ sodium pyrophosphate). The tissue was then homogenized with a glass-tissue grinder to obtain a tissue lysate, and then centrifuged for $10 \mathrm{~min}$ at $16,940 \times \mathrm{g}$ at $4{ }^{\circ} \mathrm{C}$ to remove dispensable material and collect the supernatant. Protein concentration in the samples was determined by the Bradford method using BSA as a standard [28]. Whole heart tissue extracts were obtained by immediately removing the heart after animal sacrifice and washing it in PBS solution to remove the excess of blood. Tissue was subsequently frozen at $-80^{\circ} \mathrm{C}$. At the day of the assays, heart tissue was washed again with PBS and then homogenized in a $10 \%(\mathrm{v} / \mathrm{v})$ RIPA buffer solution (150 mM sodium chloride, 1.0\% NP-40, $0.5 \%$ sodium deoxycholate, $0.1 \%$ sodium dodecyl sulphate and $50 \mathrm{mM}$ Tris, $\mathrm{pH} 8.0$ ) and centrifuged for $10 \mathrm{~min}$ at $14,000 \times \mathrm{g}$ at $4{ }^{\circ} \mathrm{C}$. The supernatant was collected and the protein concentration quantified using BSA as a standard [28]. All materials were kept on ice during the homogenization procedure.

\subsection{Western blotting analysis of selected mitochondrial and apoptotic-related proteins}

The specific protein content was determined by Western blotting. Lung tissue homogenate of each sample was diluted in Laemmli buffer (BioRad, Hercules, CA, USA) so that all samples had the same amount of protein and denatured at $90^{\circ} \mathrm{C}$ for $5 \mathrm{~min}$. Then, aliquots with equal protein content $(100 \mu \mathrm{g})$ were separated on a $12 \%$ polyacrylamide gel using sodium dodecyl sulphatepolyacrylamide gel electrophoresis (SDS-PAGE), and transferred electrophoretically to Polyvinylidene Fluoride (PVDF) membranes. Membranes were blocked for $1 \mathrm{~h}$ at room temperature in Trisbuffered saline solution (in mM: $137 \mathrm{NaCl}, 20$ Tris- $\mathrm{HCl}$; pH 7.6) containing $0.1 \%$ Tween-20 (TBS-T) containing $5 \%$ skimmed powder milk (except when BSA was used for Bcl-2 detection). The membranes were incubated overnight at $4{ }^{\circ} \mathrm{C}$ with antibodies against p53 (1:200), Bax (1:1000), Bcl-2 (1:200), ANT (1:200), VDAC (1:1000) and COX IV (1:1000), prepared in $1 \%$ skimmed powder milk (BSA for Bcl-2) in TBS-T. As control for protein loading, membranes were also probed with rabbit anti- $\beta$-actin antibody (1:5000). Following antibody incubation and washing, membranes were incubated for $1 \mathrm{~h}$ at room temperature with an alkaline phosphatase-linked secondary antibody in a dilution of 1:5000 in TBS-T containing $0.5 \%$ skimmed powder milk. The membranes were processed for detection of proteins of interest using the ECF system on the Versa Doc Imaging System and the quantification was performed using Quantity One 1-D Analysis Software Version 4.6.9, PC (BioRad). The strength of signal was expressed by calculating the difference between the average intensity of an identified area and its corresponding local background.

\subsection{Quantification of protein-bound carbonyls}

The protein reactive carbonyls in samples were analyzed according to Robinson et al. with slight modifications [29]. A determined volume of lung tissue homogenate $(\mathrm{V})$ containing $20 \mu \mathrm{g}$ of protein was derivatized with dinitrophenylhydrazine (DNPH). For this purpose, a volume ( $1 \mathrm{~V}$ ) of $12 \%$ sodium dodecyl sulphate (SDS) and two volumes $(2 \mathrm{~V})$ of $20 \mathrm{mM}$ DNPH prepared in $10 \%$ trifluoroacetic acid (TFA) was added to each sample, followed by its incubation in the dark for $5 \mathrm{~min}$. After this period, the neutralization reaction was achieved by the addition of 1.5 volumes (1.5 V) of $2 \mathrm{M}$ Tris and $18 \%$ of $\beta$-mercaptoethanol. A negative control was simultaneously prepared for each sample in the absence of DNPH. After the dilution of the samples in a Tris buffer solution to obtain a final concentration of $5 \mu \mathrm{g} / \mu \mathrm{l}, 10 \mu \mathrm{g}$ of protein was transferred to the gel for SDS-PAGE. From this point further, the procedure was equivalent to that already described for Western blotting analysis. A primary rabbit polyclonal anti-DNP antibody (1:2000) was incubated with the membrane for $2 \mathrm{~h}$ at room temperature.

\subsection{Measurement of malondialdehyde levels}

Levels of lipid peroxidation were measured by the formation of a thiobarbituric acid (TBA) adduct of malondialdehyde (MDA), separated by high-performance liquid chromatography (HPLC) [30] using an analytical column Spherisorb ODS2 $5 \mu \mathrm{m}$ 
$(250 \mathrm{~mm} \times 4.6 \mathrm{~mm})$, eluted with $60 \%(\mathrm{v} / \mathrm{v})$ potassium phosphate buffer $50 \mathrm{mM}, \mathrm{pH} 6.8$, and $40 \%(\mathrm{v} / \mathrm{v})$ methanol at a flow rate of $1 \mathrm{ml} / \mathrm{min}$. Spectrophotometric detection of the TBA-MDA adducts occurred at $532 \mathrm{~nm}$ (Gilson, Lewis Center, OH, USA). The MDA content of the samples was calculated from a standard curve prepared using the TBA-MDA complex and was expressed as nmol/mg protein.

\subsection{Measurement of reduced glutathione (GSH) and oxidized glutathione (GSSG) contents}

GSH and GSSG levels were determined by fluorescence detection after reaction of the supernatants from homogenized lung tissue containing $\mathrm{H}_{3} \mathrm{PO}_{4} / \mathrm{NaH}_{2} \mathrm{PO}_{4}-E D T A$ or $\mathrm{H}_{3} \mathrm{PO}_{4} / \mathrm{NaOH}$, respectively, with o-phthalaldehyde (OPT), pH 8.0, according to Hissin and Hilf [31]. In brief, lung tissue extracted from each treatment $(250 \mathrm{mg}$ ) was resuspended in $1.5 \mathrm{ml}$ phosphate buffer $(100 \mathrm{mM}$ $\mathrm{NaH}_{2} \mathrm{PO}_{4}, 5 \mathrm{mM}$ EDTA, pH 8.0) plus $500 \mu \mathrm{H}_{3} \mathrm{PO}_{4} 25 \%$ and was rapidly centrifuged at $135,500 \times g$ (Beckman, TL-100 ultracentrifuge) for $30 \mathrm{~min}$. For GSH determination, $100 \mu \mathrm{l}$ of supernatant of each treatment was added to $1.8 \mathrm{ml}$ phosphate buffer and $100 \mu \mathrm{l}$ OPT. For GSSG determination, $100 \mu \mathrm{l}$ of the supernatant of each treatment was added to $200 \mu$ l of $0.04 \mathrm{M}$ N-ethylmaleimide and incubated at room temperature for $30 \mathrm{~min}$. To this mixture $4.3 \mathrm{ml}$ of $0.1 \mathrm{M} \mathrm{NaOH}$ buffer and $100 \mu \mathrm{l}$ OPT was added. In both cases, after thorough mixing and incubation at room temperature for $15 \mathrm{~min}$, the solution was transferred to a quartz cuvette and the fluorescence was measured at 460 and $350 \mathrm{~nm}$ emission and excitation wavelengths, respectively. GSH and GSSG contents were determined from comparisons with a linear GSH or GSSG standard curve, respectively, and expressed as nmol/mg protein.

\subsection{Measurement of vitamin E content}

Extraction and separation of vitamin $\mathrm{E}$ ( $\alpha$-tocopherol) from lung tissue extracts were performed by following a method previously described by Vatassery and Younoszai [32]. Briefly, $1.5 \mathrm{ml}$ of SDS $(10 \mathrm{mM})$ was added to $0.5 \mathrm{mg}$ of lung tissue extract, followed by the addition of $2 \mathrm{ml}$ of ethanol. Then $2 \mathrm{ml}$ of hexane and $50 \mu \mathrm{l}$ of $3 \mathrm{M}$ $\mathrm{KCl}$ were added, and the mixture was vortexed for about $3 \mathrm{~min}$. The extract was centrifuged at $1666 \times g$ (Sorvall RT6000 refrigerated centrifuge) and $1 \mathrm{ml}$ of the upper phase, containing n-hexane, was recovered and evaporated until dryness under a stream of $\mathrm{N}_{2}$ and kept at $-80^{\circ} \mathrm{C}$. The extract was dissolved in n-hexane, and vitamin E content was analyzed by reverse-phase HPLC. A Spherisorb S10w column $(250 \mathrm{~mm} \times 4.6 \mathrm{~mm})$ was eluted with $\mathrm{n}$-hexane modified with $0.9 \%$ methanol, at a flow rate of $1.5 \mathrm{ml} / \mathrm{min}$. Detection was performed by a UV detector at $287 \mathrm{~nm}$ (Gilson, Lewis Center, $\mathrm{OH}, \mathrm{USA}$ ). The total content of vitamin $\mathrm{E}$ was calculated as nmol/mg protein.

\subsection{Determination of caspase 9- and 3-like activities}

Sample volumes corresponding to $50 \mu \mathrm{g}$ of protein for caspase 9 assays or $25 \mu \mathrm{g}$ of protein for caspase 3 assays were added to freshly prepared protease assay buffer (25 mM HEPES pH 7.5, 0.1\% CHAPS, $10 \%$ sucrose and $10 \mathrm{mM}$ dithiotreitol) to a final volume of $195 \mu \mathrm{l}$. Next, $5 \mu \mathrm{l}$ of $4 \mathrm{mM}$ substrate stock solution ( $100 \mu \mathrm{M}$ final concentration prepared in DMSO) for caspase 9 (AcLEHD-pNA) or for caspase 3 (AcDeved-pNA) was added. Reactions in the absence of lung tissue extract were used as negative controls. The samples were incubated for $2 \mathrm{~h}$ at $37^{\circ} \mathrm{C}$ and the p-nitroaniline released from caspase substrates was monitored at $405 \mathrm{~nm}$ using a spectrophotometer (Hitachi F-2500, San Jose, CA, USA). Caspase 9- and 3-like activities were expressed as [pNA released] $\mathrm{nM} / \mu \mathrm{g}$ protein.

\subsection{Statistical analysis}

Results are presented as means \pm SEM of the indicated number of experiments. A two-tailed paired Student's $t$ test was used to assess the significance of differences between two groups. Statistical analyses were performed with Graphpad Prism 5 (Graphpad Software Inc.). Differences were considered statistically significant if $p<0.05$.

\section{Results}

\subsection{DOX treatment decreases body weight without affecting lung weight}

The negative effects of DOX in the growth profile of treated rats are demonstrated by the inhibition of growth rate (data not shown) and decreased final body weight (Fig. 1A, $p<0.01$ ), although lung weight was not different when compared with saline-treated rats (Fig. 1B). Again, no differences were found when lung weight was normalized against body weight (Fig. 1C). The slight increase in this ratio is clearly due to the decrease in body weight rather than an increase in tissue weight (Fig. 1C). In the same treatment protocol, no alteration in heart weight was observed (data not shown).

\subsection{Lungs of DOX-treated rats have no detectable increase in p53/Bax signaling}

The next step was to investigate whether the sub-chronic DOX treatment results in increased expression of p53 and one of its transcriptional targets, Bax, as previously detected in cardiac cells treated in vitro with DOX [33]. No differences were observed between saline and DOX-treated rats regarding p53 (Fig. 2A) or Bax (Fig. 2B). Also, no differences between groups were observed regarding the anti-apoptotic protein $\mathrm{Bcl}-2$ or even in the Bax/Bcl-2 ratio (Fig. $2 \mathrm{C}$ and $\mathrm{D}$ ). When performing the same analysis in whole heart tissue from the same animals, we could not obtain visible bands for either p53 or Bax, regardless of the different commercial antibodies used (data not shown).

\subsection{VDAC and ANT levels are unchanged in the lung after DOX treatment}

Two important proteins for the apoptotic/necrotic process are the mitochondrial proteins adenine nucleotide translocator (ANT) and the voltage-dependent anion channel (VDAC). The data indicates that DOX treatment does not alter ANT or VDAC content in the lung (Fig. $3 \mathrm{~A}$ and $\mathrm{B}$ ).

Possible alterations in two critical oxidative phosphorylation proteins were also studied in order to obtain a crude and indirect measurement of mitochondrial mass. Two nuclear-encoded subunits, NDUFS3 and COX IV, which are part of NADH-coenzyme $\mathrm{Q}$ reductase (complex I) and cytochrome c oxidase (complex IV), respectively, were selected. As seen in Fig. $3 C$ and D, no significant alterations were observed in the two subunits after DOX treatment.

\subsection{DOX causes lung oxidative stress as seen by increased MDA formation and vitamin E content decrease}

Direct reaction of proteins with ROS can lead to the formation of protein derivatives or peptide fragments possessing highly reactive carbonyl groups, including ketones and aldehydes [29]. No increase of oxidized proteins in the lungs of DOX-treated rats was observed (Fig. 4A and B). The presence of oxidized proteins in both groups is confirmed by the specificity of DNPH (Fig. 4A and B). To determine lipid peroxidation in the lungs of DOX-treated rats malondialdehyde (MDA), a marker of lipid peroxidation, was measured. 

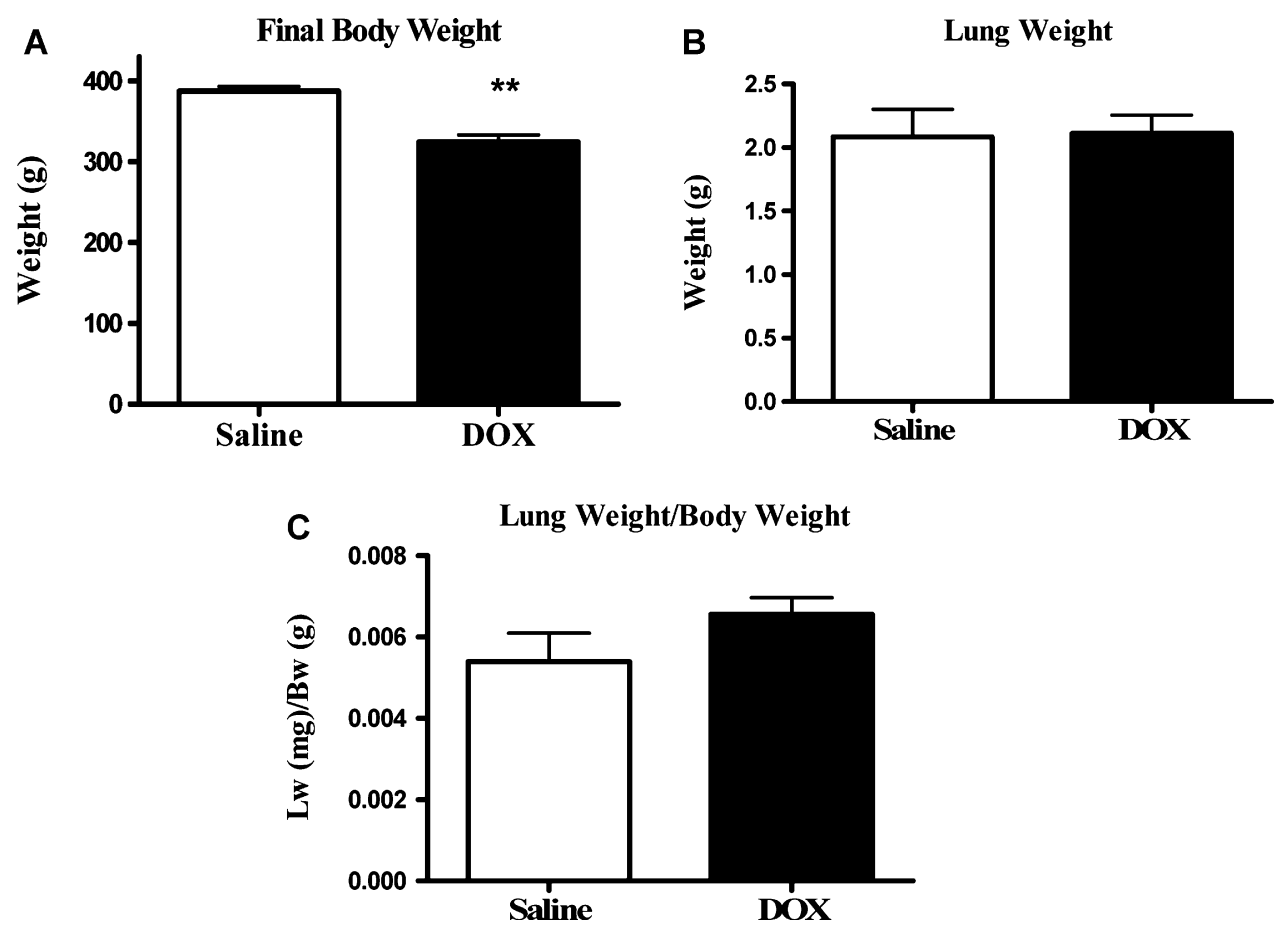

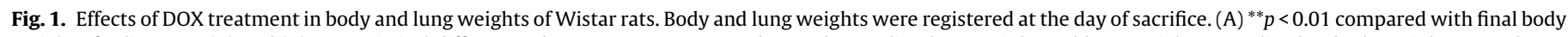

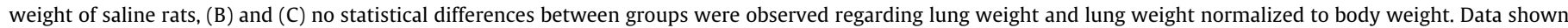
represents mean \pm SEM from 8 to 10 different animals per group.
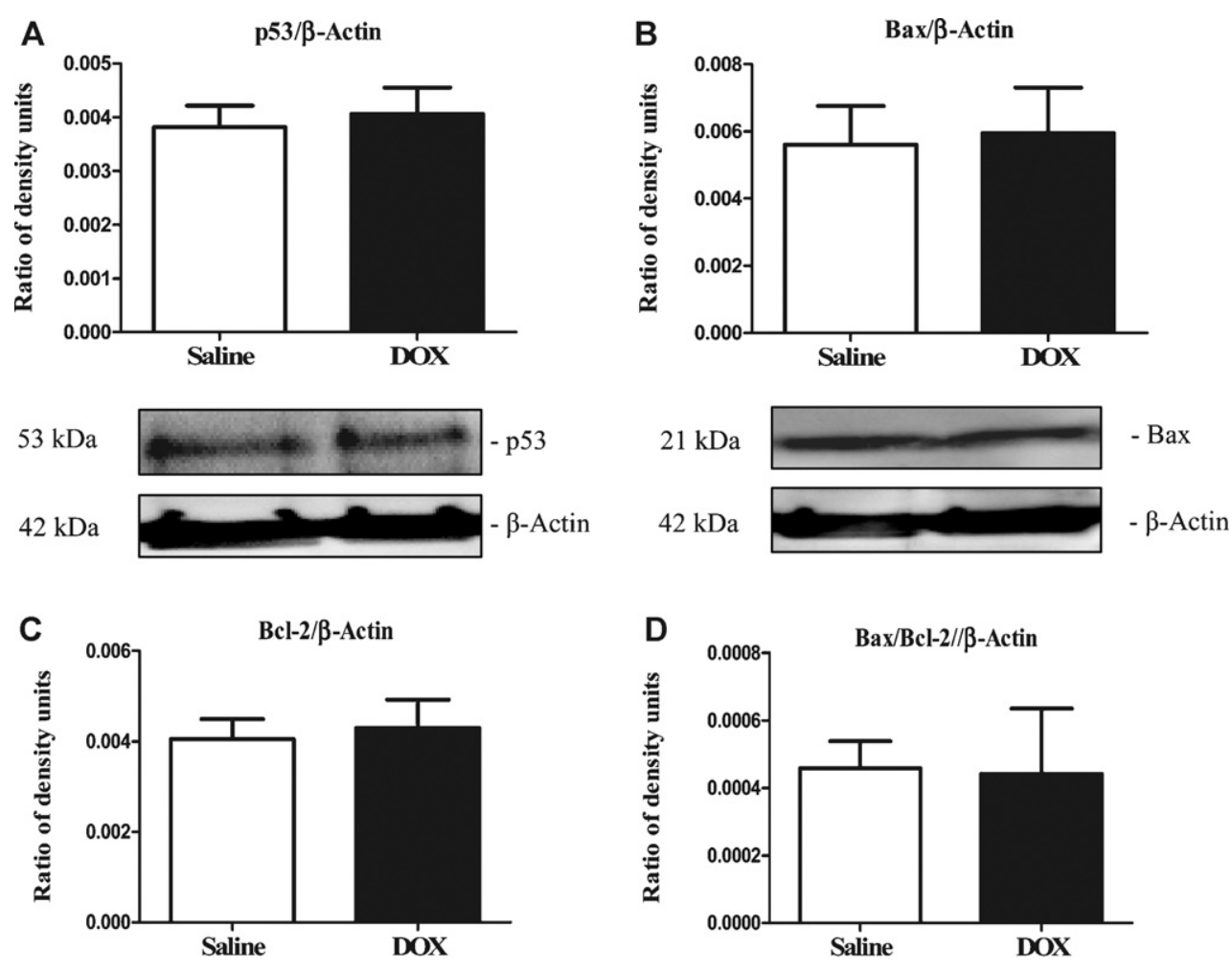

$26 \mathrm{kDa}$

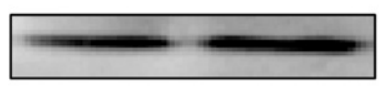

$-\mathrm{Bcl}-2$

$42 \mathrm{kDa}$

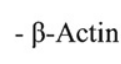

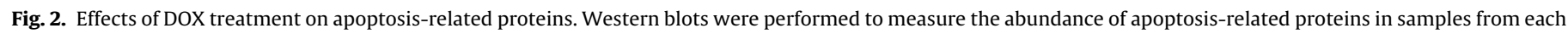

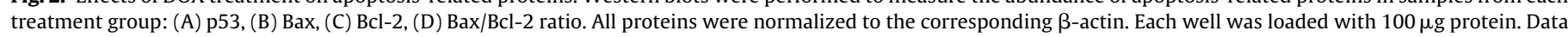
shown represents mean \pm SEM from 8 to 10 different animals per group. 

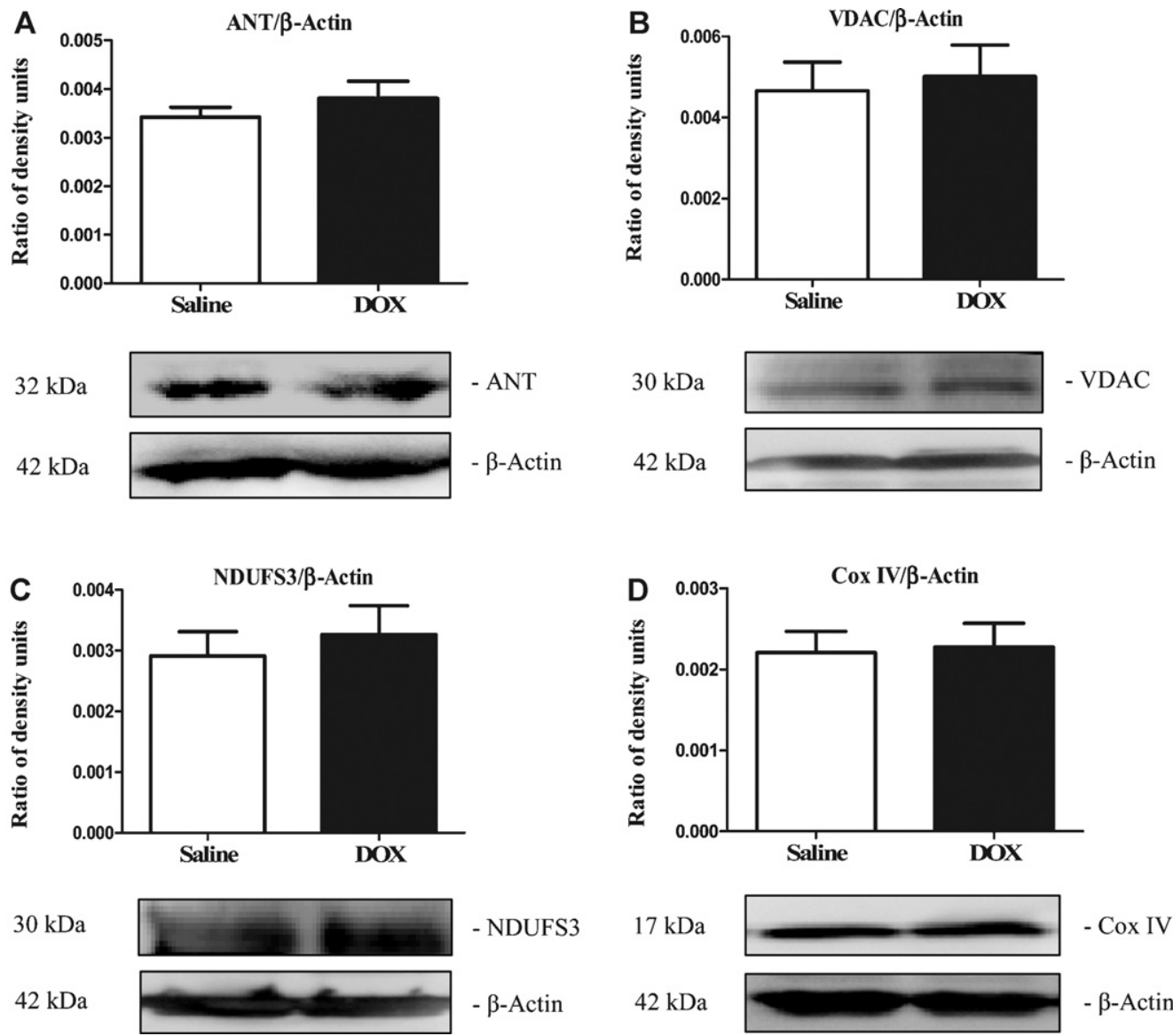

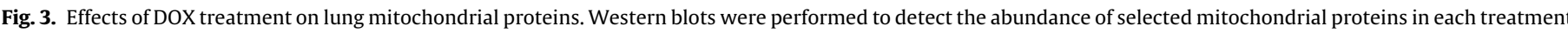

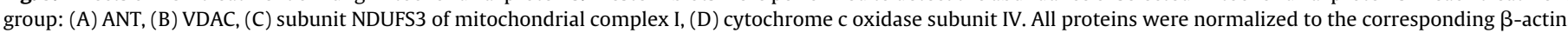
Each well was loaded with $100 \mu \mathrm{g}$ protein. Data shown represents mean \pm SEM from 8 to 10 different animals per group.

A significant difference $(p<0.05)$ was observed between the two groups (Fig. 4C). Lung homogenates obtained from DOX-treated animals presented no significant differences in GSH or GSSG levels when compared with control animals (Fig. 4D). Interestingly, subchronic DOX treatment induced a significant decrease in vitamin E levels $(p<0.001)$ compared with saline-treated rats (Fig. 4E). By using whole heart tissue obtained from the same animals, cardiac MDA levels and vitamin E content showed no statistical significant differences between the two groups (Fig. 4C and E).

\subsection{DOX treatment does not increase caspase 9- and 3-like activities}

To confirm the absence of apoptotic signaling in the lung after DOX treatment, the next step was to measure caspases 9- and 3-like activities. The data obtained revealed no increase in either caspaselike activities in the lungs of DOX-treated rats, as compared to saline rats (Fig. 5). Interestingly, a significant increase in caspase 3 and caspase 9-like activities was observed in the hearts of the same experimental animals (Fig. 5).

\section{Discussion}

Although DOX-induced cardiotoxicity has been widely studied, pulmonary toxicity has been scarcely investigated, although several cases have been previously reported [34-37].

After a sub-chronic treatment protocol, DOX caused a decrease in both the growth rate (data not shown) and in the body weight at the time of sacrifice (Fig. 1). Baciewicz et al. [27] proposed that the loss of weight gain can be correlated with the distress of the injection procedure due to the burning and caustic effects of DOX leading to hematological and gastrointestinal disturbances, being one of the many factors affecting growth and final body weight. Nevertheless, the ratio between lung and body weight was not altered (Fig. 1)

It has been previously described that DoX activates the p53/Bax axis in cultured cardiomyoblasts [33]. Interestingly, it has been proposed that the intracellular localization of p53 is regulated through redox-dependent mechanisms, which are dependent upon DOX pro-oxidant activity [38]. In fact, it has also been previously demonstrated in different experimental models that DOX treatment results in increased mitochondrial p53/Bax content increased cellular Bcl-2/Bax ratio, cytochrome c release from mitochondria, caspase 3 activation, DNA fragmentation and apoptosis $[33,39]$. In this study, and under our treatment protocol, total p53, Bax and Bcl-2, as well as the ratio between Bcl-2 and Bax was not increased in lung after DOX treatment (Fig. 2).

The results suggest that for the treatment used, no increased pro- or anti-apoptotic signaling occurred. Since p53 is involved in DNA damage response, contributing to the regulation of Bax, it can be concluded that (a) DOX does not cause lung nuclear DNA damage or (b) nuclear DNA damage occurred in an early time point and was repaired. No detectable p53/Bax was observed in the hearts from the same animals, regardless of the treatment group (data not shown).

The absence of apoptotic signaling in the lung is confirmed by unaltered caspase 3 and caspase 9-like activities (Fig. 5). The lack of significant differences does not exclude the possibility that DOX 
A

$250 \mathrm{kDa}$ -

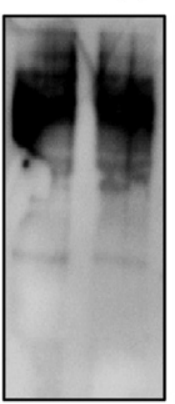

Saline DOX
DNPH (-)

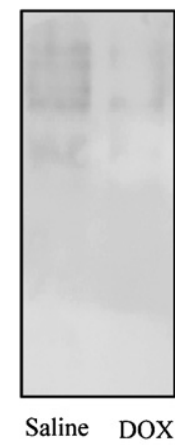

C

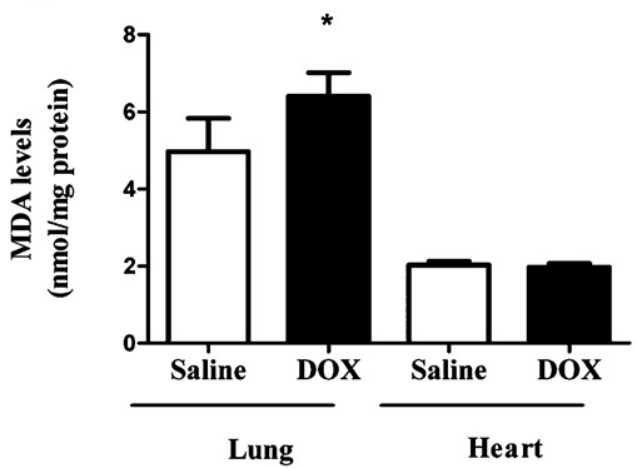

B

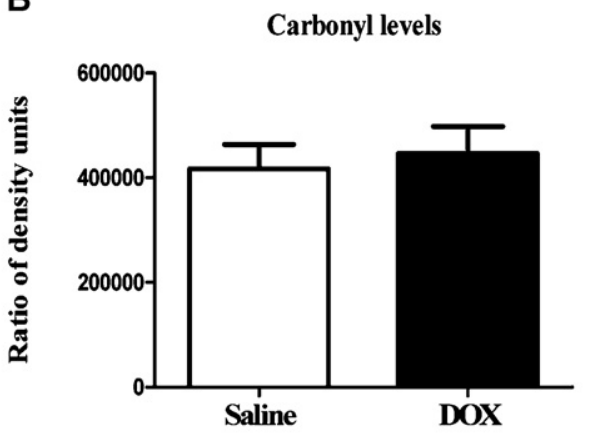

D

Glutathione content
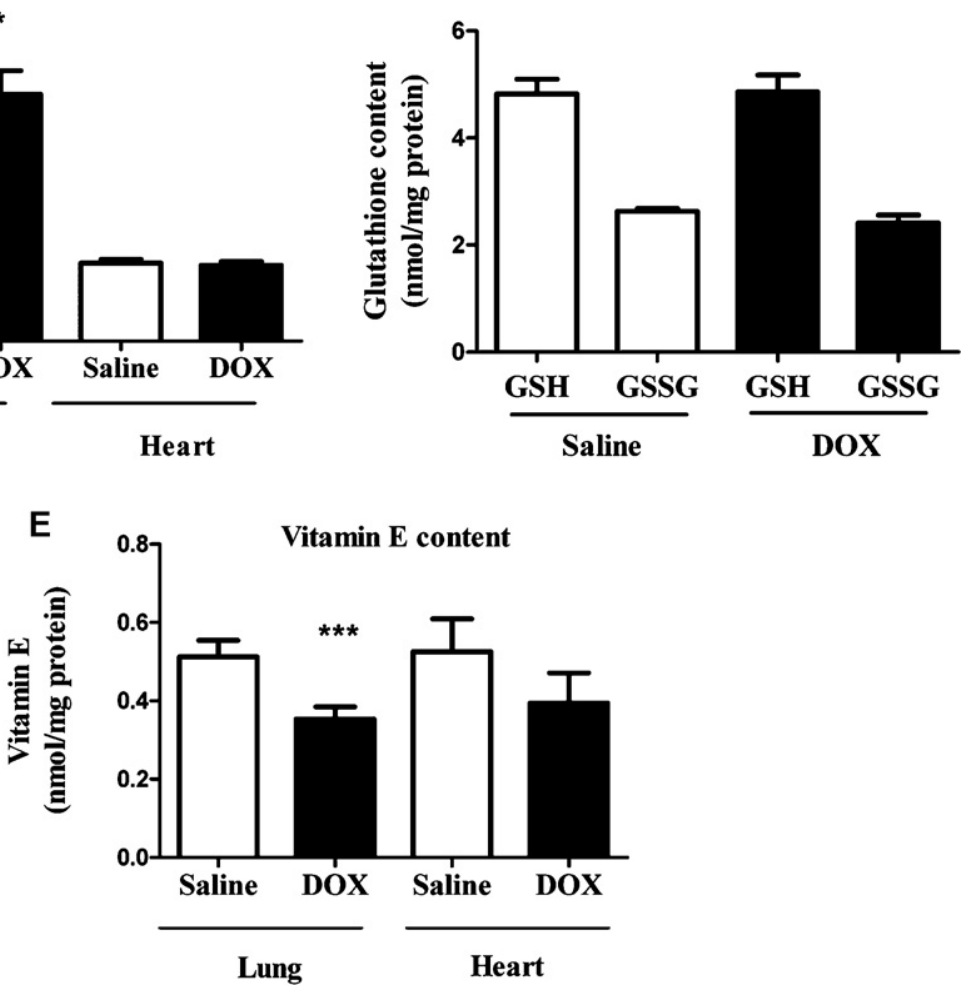

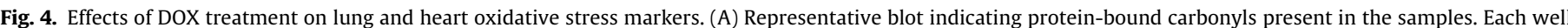

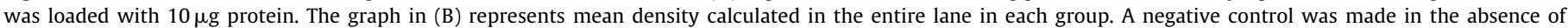

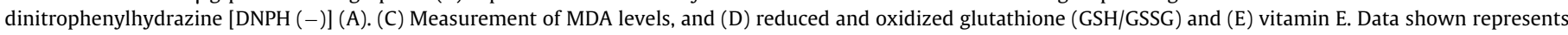
mean \pm SEM from 8 to 10 different animals per group. Statistical significance: ${ }^{* * *} p<0.001$; ${ }^{*} p<0.05$ compared with control lung tissue.
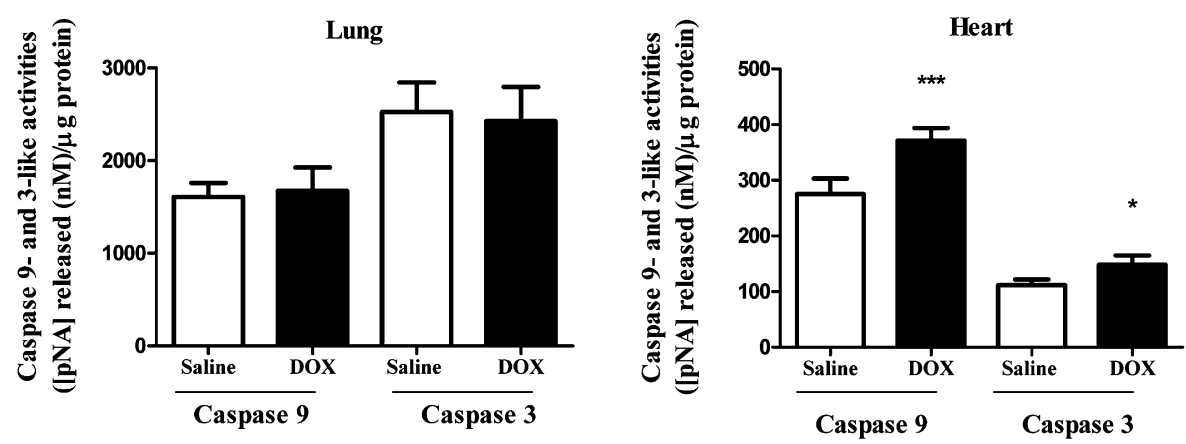

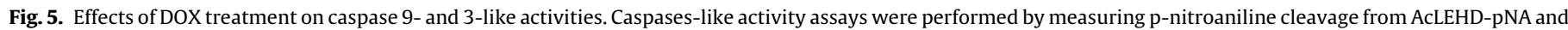

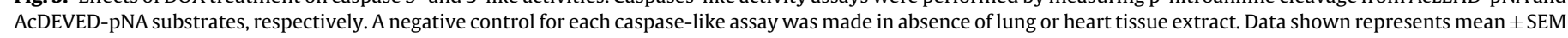
from 8 to 10 different animals per group. Statistical significance: ${ }^{* * *} p<0.001 ;{ }^{*} p<0.05$ compared with control heart tissue. 
treatment resulted in an initial increase in caspase activation, and consequent apoptosis, although no loss of lung mass was observed. In contrast to caspase lung data, heart samples from the same animals revealed an increase in both caspase 3 and 9-like activities (Fig. 5). The differences indicate an organ-specific apoptotic signaling that is triggered by DOX, although the functional consequences of increased caspase activity in the heart are not immediately clear.

Several lung mitochondrial proteins were analyzed by Western blotting in order to identify whether DOX would cause alterations in the mitochondrial content of those proteins. Two of those proteins were the ANT and the VDAC, two possible components of the MPT pore and which have been implicated in cell death and mitochondrial homeostasis [40]. In a previous work, our group demonstrated that the same treatment protocol in a different rat strain resulted in a decrease of ANT in the hearts of DOX-treated rats [18]. In the present work, the results show that lung VDAC and ANT remain unchanged after DOX treatment (Fig. 3A and B).

Another possible consequence of DOX treatment would be a decreased expression of some mitochondrial respiratory chain proteins. Two selected oxidative phosphorylation proteins were studied, NDUFS3 and COX IV in order to investigate not only possible DOX effects in their mitochondrial content, but also to have a crude and indirect estimate of mitochondrial mass. NDUFS3 is a core subunit of complex I that is believed to belong to the minimal assembly unit required for catalysis [41]. Complex I participates in the metabolic conversion of DOX to a reactive semi-quinone [20], which leads to increased mitochondrial oxidative stress [21] and consequent protein oxidation [23]. It has also been demonstrated that DOX downregulates the expression of critical proteins of the mitochondrial respiratory chain, including cytochrome c oxidase subunits [42]. Interestingly, there is evidence that suggests that compromised cytochrome c oxidase subunit IV expression may result in altered sensitization to apoptosis [43]. In the present work, no differences were found in NDUFS3 or COX IV content in the lungs (Fig. $3 \mathrm{C}$ and D), although we cannot exclude the possibility that the expression of other oxidative phosphorylation proteins may be affected by DOX. This is particularly relevant in the case of mitochondrial DNA-encoded subunits, as DOX was already demonstrated to oxidize mitochondrial DNA [44].

We have previously shown that DOX causes protein oxidation in the heart, as measured by an increase in protein carbonyl groups $[16,45]$. In the present work, the sub-chronic DOX treatment does not result in enhanced lung protein carbonylation (Fig. 4A and B). In contrast, we have consistently measured an increase in MDA and a decrease in vitamin $\mathrm{E}$ in the lungs of treated animals (Fig. $4 \mathrm{C}$ and E). MDA is a classic marker of lipid peroxidation, the interaction of which with DNA and proteins has often been referred to as potentially mutagenic and atherogenic [46]. The data shows that sub-chronic DOX treatment potentiates lipid peroxidation in the lungs of treated animals. Vitamin $\mathrm{E}$ is a lipid soluble chain-breaking antioxidant found in the plasma and tissues, contributing also to the structural integrity of biological membranes [47]. Demonstrating the important role of vitamin $\mathrm{E}$ in the maintenance of membrane integrity, vitamin E concentration in different tissues is inversely correlated with lipid peroxidation [48]. The decrease in vitamin E levels shown after DOX treatment could contribute to the increase in lipid peroxidation promoted by DOX treatment (Fig. 4E).

Growing evidence suggests that GSH, a nonenzymatic antioxidant, plays an important role in the maintenance of cell redox homeostasis. GSH is a cofactor of several detoxifying enzymes and is able to regenerate important antioxidants, including vitamin $\mathrm{E}$ [49]. Interestingly, GSH and GSSG content (Fig. 4D) as well as the GSH/GSSG redox ratio (data not shown) were not different between groups. One would expect that the data regarding MDA and vitamin $\mathrm{E}$ would be associated with a decrease in GSH, demonstrating increased pro-oxidant environment in the cell. From the results, we can hypothesize that the schedule of DOX treatment did not necessarily exhaust the lung GSH pool or that enhanced GSH synthesis accompanied by GSSG export occurred, in an attempt to counteract lipid peroxidation. Another relevant possibility that can explain the decrease in vitamin $\mathrm{E}$ is that DOX-treated rats can suffer from gastrointestinal disorders [27], which may lead to a decreased absorption of dietary lipids and with that, vitamin $\mathrm{E}$ availability, since this lipid cannot be synthesized by animals and therefore originates from the diet [50]. This could also explain why GSH and GSSG levels, as well as protein carbonyls were not dissimilar from the control animals. Heart MDA and vitamin E were measured in the same animals. Interestingly, no alterations were observed (Fig. 4C and E), although the average value for vitamin $\mathrm{E}$ was lower in DOX-treated animals ( $p=0.07$ ) (Fig. 4E). As described above, it has been demonstrated that DOX causes oxidative stress in the heart $[4,16,51,52]$ and, therefore, increased levels of MDA and decreased vitamin E content would be expected. The fact that these effects were not observed in the heart may suggest that DOXinduced oxidative stress was counteracted by antioxidant/repair mechanisms, which would be more ineffective in the lung. Previous studies indicated a decrease in vitamin $\mathrm{E}$ and an increase in MDA in the hearts of DOX-treated Sprague-Dawley rats using the same treatment regimen [52]. Since the treatment protocol was the same, the conflicting results may be explained by differences between rat strains, which were previously observed $[53,54]$.

Leaving rat strain differences apart, the present work shows different consequences of DOX treatment in the heart and lung. Lipid peroxidation and decreased vitamin $\mathrm{E}$ in the lungs of DOX-treated rats can result in several consequences. For example, it has been demonstrated that decreased vitamin $\mathrm{E}$ and consequent oxidative stress increase allergic airways responses in mice [55], although in humans, vitamin E levels were not associated with changes of lung function over time in a pulmonary toxicity study [56].

The present study demonstrates that sub-chronic DOX treatment does not result in apoptotic signaling but leads to oxidative stress in the lungs, although at the moment we do not know if oxidative stress is a primary event or is instead secondary to other effects. Pulmonary toxicity of DOX is not a common side effect of chemotherapy, but it can be devastating if added to the cardiotoxicity associated with DOX therapy, possibly contributing to a lethal outcome. The pulmonary toxicity observed in some cases of DOX chemotherapy can be a phenomenon that possibly only occurs when a patient undergoes high-dose chemotherapy [57].

The results from the present work suggest that the lung and the heart differ concerning sub-chronic DOX-induced toxicity. Several reasons may explain organ-specific differences including (a) lower DOX accumulation in the lung, (b) higher antioxidant defenses, (c) higher regenerative capacity of the tissue or (d) lower DOX activation which may be explained by decreased lung mitochondrial content. Over longer treatment regimens, it cannot be excluded that cumulative DOX toxicity is present in the lung, similar to what is proposed to occur the heart [58], thus leading to a slow eroding of lung capacity.

A better understanding of DOX-induced toxicity in the cardiopulmonary system can contribute to a better management of DOX toxic effects, thus improving the therapeutic efficiency of DOX. Further studies in isolated lung mitochondria using the same or different DOX treatment protocols and in vitro pulmonary cells exposed to DOX would allow for further insights into the origins and consequences of DOX toxicity in the lung.

\section{Conflict of interest statement}

None of the authors have any conflict of interest. 


\section{Acknowledgements}

The present work was supported by Fundação para a Ciência e Tecnologia research grants (PTDC-SAU-OSM-64084-2006 and PTDC/SAU-OSM/104731/2008) and Ph.D. fellowships SFRH-BD36938-2007 to GP and SFRH-BD-64247-2009 to SP. We also thank Vilma A. Sardão, Ph.D., Maria S. Santos, Ph.D. and Ana C. Moreira (Center for Neuroscience and Cell Biology, Coimbra, Portugal) for their overall guidance and assistance in some of the techniques included in this work. We are also very thankful to Kendall B. Wallace, Ph.D. (University of Minnesota-Duluth, USA) for proofreading the present manuscript.

\section{References}

[1] C. Carvalho, R.X. Santos, S. Cardoso, S. Correia, P.J. Oliveira, M.S. Santos, P.I Moreira, Doxorubicin: the good, the bad and the ugly effect, Curr. Med. Chem. 16 (25) (2009) 3267-3285.

[2] R. Injac, B. Strukelj, Recent advances in protection against doxorubicin-induced toxicity, Technol. Cancer Res. Treat. 7 (6) (2008) 497-516.

[3] M.M. Abu-Khalaf, L. Harris, Anthracycline-induced cardiotoxicity: risk assessment and management, Oncology (Williston Park) 23 (3) (2009), 239, 244, 252

[4] E. Barry, J.A. Alvarez, R.E. Scully, T.L. Miller, S.E. Lipshultz, Anthracyclineinduced cardiotoxicity: course, pathophysiology, prevention and management Expert Opin. Pharmacother. 8 (8) (2007) 1039-1058.

[5] G. Takemura, H. Fujiwara, Doxorubicin-induced cardiomyopathy from the cardiotoxic mechanisms to management, Prog. Cardiovasc. Dis. 49 (5) (2007) 330-352.

[6] J.C. Reed, M. Pellecchia, Apoptosis-based therapies for hematologic malignancies, Blood 106 (2) (2005) 408-418.

[7] U. Fischer, K. Schulze-Osthoff, Apoptosis-based therapies and drug targets, Cell Death Differ. 12 (Suppl. 1) (2005) 942-961.

[8] J.C. Reed, Apoptosis-based therapies, Nat. Rev. Drug Discov. 1 (2) (2002) 111-121.

[9] S. Elmore, Apoptosis: a review of programmed cell death, Toxicol. Pathol. 35 (4) (2007) 495-516.

[10] V.A. Sardao, S.L. Pereira, P.J. Oliveira, Drug-induced mitochondrial dysfunction in cardiac and skeletal muscle injury, Expert Opin. Drug Saf. 7 (2) (2008) 129-146.

[11] S. Wu, Y.S. Ko, M.S. Teng, Y.L. Ko, L.A. Hsu, C. Hsueh, Y.Y. Chou, C.C. Liew, Y.S. Lee Adriamycin-induced cardiomyocyte and endothelial cell apoptosis: in vitro and in vivo studies, J. Mol. Cell. Cardiol. 34 (12) (2002) 1595-1607.

[12] L. Wang, W. Ma, R. Markovich, J.W. Chen, P.H. Wang, Regulation of cardiomyocyte apoptotic signaling by insulin-like growth factor I, Circ. Res. 83 (5) (1998) 516-522.

[13] V.A. Sardao, P.J. Oliveira, J. Holy, C.R. Oliveira, K.B. Wallace, Morphological alterations induced by doxorubicin on $\mathrm{H} 9 \mathrm{c} 2$ myoblasts: nuclear, mitochondrial, and cytoskeletal targets, Cell Biol. Toxicol. 25 (3) (2009) 227-243.

[14] S. Zhou, A. Starkov, M.K. Froberg, R.L. Leino, K.B. Wallace, Cumulative and irreversible cardiac mitochondrial dysfunction induced by doxorubicin, Cance Res. 61 (2) (2001) 771-777.

[15] K.B. Wallace, Adriamycin-induced interference with cardiac mitochondrial calcium homeostasis, Cardiovasc. Toxicol. 7 (2) (2007) 101-107.

[16] P.J. Oliveira, J.A. Bjork, M.S. Santos, R.L. Leino, M.K. Froberg, A.J. Moreno, K.B. Wallace, Carvedilol-mediated antioxidant protection against doxorubicininduced cardiac mitochondrial toxicity, Toxicol. Appl. Pharmacol. 200 (2) (2004) 159-168.

[17] L.E. Solem, T.R. Henry, K.B. Wallace, Disruption of mitochondrial calcium homeostasis following chronic doxorubicin administration, Toxicol. Appl. Pharmacol. 129 (2) (1994) 214-222.

[18] P.J. Oliveira, K.B. Wallace, Depletion of adenine nucleotide translocator protein in heart mitochondria from doxorubicin-treated rats-relevance for mitochondrial dysfunction, Toxicology 220 (2-3) (2006) 160-168.

[19] R.D. Olson, P.S. Mushlin, Doxorubicin cardiotoxicity: analysis of prevailing hypotheses, FASEB J. 4 (13) (1990) 3076-3086.

[20] K.B. Wallace, Doxorubicin-induced cardiac mitochondrionopathy, Pharmacol Toxicol. 93 (3) (2003) 105-115.

[21] Y. Zhang, O. Marcillat, C. Giulivi, L. Ernster, K.J. Davies, The oxidative inactivation of mitochondrial electron transport chain components and ATPase, J. Biol. Chem. 265 (27) (1990) 16330-16336.

[22] J. Goodman, P. Hochstein, Generation of free radicals and lipid peroxidation by redox cycling of adriamycin and daunomycin, Biochem. Biophys. Res. Commun. 77 (2) (1977) 797-803.

[23] E.J. Demant, Inactivation of cytochrome c oxidase activity in mitochondrial membranes during redox cycling of doxorubicin, Biochem. Pharmacol. 41 (4) (1991) 543-552.

[24] R.F. Vidal, S. Eksborg, M. Sundberg, M. Carlberg, B. Elfsson, B.S. Andersson, Doxorubicin- and daunorubicin-induced energy deprivation and nucleotide degradation in isolated cardiomyocytes, Toxicology 114 (1) (1996) 1-10.
[25] M. Meadors, J. Floyd, M.C. Perry, Pulmonary toxicity of chemotherapy, Semin. Oncol. 33 (1) (2006) 98-105.

[26] R.F. Minchin, M.R. Johnston, H.M. Schuller, M.A. Aiken, M.R. Boyd, Pulmonary toxicity of doxorubicin administered by in situ isolated lung perfusion in dogs, Cancer 61 (7) (1988) 1320-1325.

[27] F.A. Baciewicz Jr., M. Arredondo, B. Chaudhuri, K.A. Crist, D. Basilius, S. Bandyopadhyah, N.R. Thomford, P.K. Chaudhuri, Pharmacokinetics and toxicity of isolated perfusion of lung with doxorubicin, J. Surg. Res. 50 (2) (1991) 124-128.

[28] M.M. Bradford, A rapid and sensitive method for the quantitation of microgram quantities of protein utilizing the principle of protein-dye binding, Anal. Biochem. 72 (1976) 248-254.

[29] C.E. Robinson, A. Keshavarzian, D.S. Pasco, T.O. Frommel, D.H. Winship, E.W. Holmes, Determination of protein carbonyl groups by immunoblotting, Anal. Biochem. 266 (1) (1999) 48-57.

[30] H.H. Draper, M. Hadley, Malondialdehyde determination as index of lipid peroxidation, Methods Enzymol. 186 (1990) 421-431.

[31] P.J. Hissin, R. Hilf, A fluorometric method for determination of oxidized and reduced glutathione in tissues, Anal. Biochem. 74 (1) (1976) 214-226.

[32] G.T. Vatassery, R. Younoszai, Alpha tocopherol levels in various regions of the central nervous systems of the rat and guinea pig, Lipids 13 (11) (1978) 828-831.

[33] V.A. Sardao, P.J. Oliveira, J. Holy, C.R. Oliveira, K.B. Wallace, Doxorubicininduced mitochondrial dysfunction is secondary to nuclear p53 activation in H9c2 cardiomyoblasts, Cancer Chemother. Pharmacol. (2009).

[34] H. Bredenfeld, J. Franklin, L. Nogova, A. Josting, S. Fries, V. Mailander, J. Oertel, V. Diehl, A. Engert, Severe pulmonary toxicity in patients with advanced-stage Hodgkin's disease treated with a modified bleomycin, doxorubicin, cyclophosphamide, vincristine, procarbazine, prednisone, and gemcitabine (BEACOPP) regimen is probably related to the combination of gemcitabine and bleomycin: a report of the German Hodgkin's Lymphoma Study Group, J. Clin. Oncol. 22 (12) (2004) 2424-2429.

[35] J.W. Friedberg, D. Neuberg, H. Kim, S. Miyata, M. McCauley, D.C. Fisher, T. Takvorian, G.P. Canellos, Gemcitabine added to doxorubicin, bleomycin, and vinblastine for the treatment of de novo Hodgkin disease: unacceptable acute pulmonary toxicity, Cancer 98 (5) (2003) 978-982.

[36] J.Verschoore, J.L. Lagrange, J.L. Boublil, J.M. Aubanel, B. Blaive, J. Pinto, M. Namer, Pulmonary toxicity of a combination of low-dose doxorubicin and irradiation for inoperable lung cancer, Radiother. Oncol. 9 (4) (1987) 281-288.

[37] I. Dimopoulou, A. Bamias, P. Lyberopoulos, M.A. Dimopoulos, Pulmonary toxicity from novel antineoplastic agents, Ann. Oncol. 17 (3) (2006) 372-379.

[38] R. Nithipongvanitch, W. Ittarat, M.P. Cole, J. Tangpong, D.K. Clair, T.D. Oberley, Mitochondrial and nuclear p53 localization in cardiomyocytes: redox modulation by doxorubicin (Adriamycin)? Antioxid. Redox Signal. 9 (7) (2007) 1001-1008.

[39] A.C. Childs, S.L. Phaneuf, A.J. Dirks, T. Phillips, C. Leeuwenburgh, Doxorubicin treatment in vivo causes cytochrome $C$ release and cardiomyocyte apoptosis, as well as increased mitochondrial efficiency, superoxide dismutase activity, and Bcl-2:Bax ratio, Cancer Res. 62 (16) (2002) 4592-4598.

[40] N. Zamzami, G. Kroemer, The mitochondrion in apoptosis: how Pandora's box opens, Nat. Rev. Mol. Cell Biol. 2 (1) (2001) 67-71.

[41] R.O. Vogel, C.E. Dieteren, L.P. van den Heuvel, P.H. Willems, J.A. Smeitink, W.J. Koopman, L.G. Nijtmans, Identification of mitochondrial complex I assembly intermediates by tracing tagged NDUFS3 demonstrates the entry point of mitochondrial subunits, J. Biol. Chem. 282 (10) (2007) 7582-7590.

[42] K. Chandran, D. Aggarwal, R.Q. Migrino, J. Joseph, D. McAllister, E.A. Konorev W.E. Antholine, J. Zielonka, S. Srinivasan, N.G. Avadhani, B. Kalyanaraman, Doxorubicin inactivates myocardial cytochrome c oxidase in rats: cardioprotection by Mito-Q, Biophys. J. 96 (4) (2009) 1388-1398.

[43] Y. Li, J.S. Park, J.H. Deng, Y. Bai, Cytochrome c oxidase subunit IV is essential for assembly and respiratory function of the enzyme complex, J. Bioenergy Biomembr. 38 (5-6) (2006) 283-291.

[44] C.M. Palmeira, J. Serrano, D.W. Kuehl, K.B. Wallace, Preferential oxidation of cardiac mitochondrial DNA following acute intoxication with doxorubicin, Biochim. Biophys. Acta 1321 (2) (1997) 101-106.

[45] J.M. Berthiaume, P.J. Oliveira, M.W. Fariss, K.B. Wallace, Dietary vitamin E decreases doxorubicin-induced oxidative stress without preventing mitochondrial dysfunction, Cardiovasc. Toxicol 5 (3) (2005) 257-267.

[46] D. Del Rio, A.J. Stewart, N. Pellegrini, A review of recent studies on malondialdehyde as toxic molecule and biological marker of oxidative stress, Nutr. Metab. Cardiovasc. Dis. 15 (4) (2005) 316-328.

[47] G.W. Burton, M.G. Traber, Vitamin E: antioxidant activity, biokinetics, and bioavailability, Annu. Rev. Nutr. 10 (1990) 357-382.

[48] D.J. Kornbrust, R.D. Mavis, Relative susceptibility of microsomes from lung, heart, liver, kidney, brain and testes to lipid peroxidation: correlation with vitamin E content, Lipids 15 (5) (1980) 315-322.

[49] M. Valko, D. Leibfritz, J. Moncol, M.T. Cronin, M. Mazur, J. Telser, Free radicals and antioxidants in normal physiological functions and human disease, Int. J. Biochem. Cell Biol. 39 (1) (2007) 44-84.

[50] C. Villaverde, L. Cortinas, A.C. Barroeta, S.M. Martin-Orue, M.D. Baucells, Relationship between dietary unsaturation and vitamin E in poultry, J. Anim. Physiol. Anim. Nutr. (Berl.) 88 (3-4) (2004) 143-149.

[51] J.M. Berthiaume, K.B. Wallace, Adriamycin-induced oxidative mitochondrial cardiotoxicity, Cell Biol. Toxicol. 23 (1) (2007) 15-25.

[52] P.J. Oliveira, M.S. Santos, K.B. Wallace, Doxorubicin-induced thiol-dependent alteration of cardiac mitochondrial permeability transition and respiration, Biochemistry (Mosc.) 71 (2) (2006) 194-199. 
[53] N. Hlavacova, J. Bakos, D. Jezova, Differences in home cage behavior and endocrine parameters in rats of four strains, Endocr. Regul. 40 (4) (2006) $113-118$.

[54] E.R. Kuhn, K. Bellon, L. Huybrechts, W. Heyns, Endocrine differences between the Wistar and Sprague-Dawley laboratory rat: influence of cold adaptation, Horm. Metab. Res. 15 (10) (1983) 491-498.

[55] M. Talati, B. Meyrick, R.S. Peebles Jr., S.S. Davies, R. Dworski, R. Mernaugh, D. Mitchell, M. Boothby, L.J. Roberts 2nd, J.R. Sheller, Oxidant stress modulates murine allergic airway responses, Free Radic. Biol. Med. 40 (7) (2006) 1210-1219.
[56] H.S. Alfonso, L. Fritschi, N.H. de Klerk, G. Ambrosini, J. Beilby, N. Olsen, A.W Musk, Plasma retinol, carotene and vitamin E concentrations and lung function in a crocidolite-exposed cohort from Wittenoom. Western Australia: a cohort study, Nutr. J. 4 (2005) 16.

[57] B.E. Brockstein, C. Smiley, J. Al-Sadir, S.F. Williams, Cardiac and pulmonary toxicity in patients undergoing high-dose chemotherapy for lymphoma and breas cancer: prognostic factors, Bone Marrow Transplant. 25 (8) (2000) 885-894.

[58] D. Lebrecht, B. Setzer, U.P. Ketelsen, J. Haberstroh, U.A. Walker, Time-dependent and tissue-specific accumulation of mtDNA and respiratory chain defects in chronic doxorubicin cardiomyopathy, Circulation 108 (19) (2003) 2423-2429. 